\title{
Polymer-Doped UHF RFID Tag for Wireless-Sensing of Humidity
}

\author{
S.Manzari*, C. Occhiuzzi*, S. Nawale ${ }^{\dagger}$, A. Catini ${ }^{\ddagger}$, C. Di Natale ${ }^{\ddagger}$, and G. Marrocco* \\ * DISP, University of Roma Tor Vergata, \\ Via del Politecnico, 1, 00133, Roma (ITALY) \\ † Veermata Jijabai Technological Institute, \\ Mumbay, India \\ $\ddagger$ ELN, University of Roma Tor Vergata, \\ Via del Politecnico, 1, 00133, Roma (ITALY)
}

\begin{abstract}
Passive UHF RFID tags, beside item labelling, are also able to exploit capability of sensing the physical state of the tagged object as well as of the surrounding environment. Here a family of polymer-doped tags are proposed and fully characterized for the detection of ambient humidity. A sensitive chemical species based on PEDOT:PSS is used to dope a properly shaped slot carved into a folded-like patch tag. The communication and sensing capabilities of the radio-sensor are investigated by means of simulation and measurements showing how to control and balance above opposite requirements by dosing the quantity of sensitive material. The device could have interesting application in the assessment of the air quality in living and controlled rooms, in the monitoring of the conservation state of foods, in the preservation of walls, and even to monitor the healing degree of wounds.
\end{abstract}

Index Terms-RFID, Sensor, humidity, polymer, PEDOT:PSS

\section{INTRODUCTION}

The use of passive Radio Frequency IDentification (RFID) tags as sensors [1] has been very recently investigated for the wireless observation of several processes in evolution. In particular, sensitive materials may be integrated together with the tag's antenna at the purpose to transduce chemical/physical variation into changes of the tags' radiation performances. Some preliminary experiments with dipoles loaded by carbon nanotubes demonstrated the possibility to sense the presence of toxic gas in the air [2], while the use of shape-memory alloys enabled the threshold observation of environmental temperature [3]. It is hence feasible to imagine many applications to the observation of the environmental conditions by pervasively distributed low-cost sensors within houses, warehouses and workplaces in general.

Among different environmental parameters, the observation of humidity is of primarily interest in the assessment of the air quality in living and controlled rooms, in the monitoring of the conservation state of foods, in the preservation of walls, exhibition spaces, hystorical buildings, libraries and archival collections. Not last, moisture is critical in many biomedical applications concerning the healing degree of wounds and drug delivery in general.

Some early experiments on how integrating humidity sensors into RFID tags have been very recently presented for UHF passive applications. In [4] the sensitive material was simply a blotting paper, eventually doped with $\mathrm{NaCl}$ (salt), covering an RFID patch-like tag. Since the paper absorbs water, the radiation performances of the tag sensibly degrade, thus producing appreciable variations of the tag's response. An inkjet printed tag over a Kapton substrate was instead experimented in [5]. The sensitivity and the communication performances have been evaluated in term of turn-on power and frequency shift.

In these papers, however, no attempt was done to master the achievable sensitivity and to characterize the dynamic response of such class of devices.

This work explores for the first time the possibility to integrate polymeric species into a passive UHF tag taking into account both the communication and sensing issues. These materials, as discussed later on, have been already used as humidity sensors with interesting performances, but only within DC systems and never applied to UHF RFIDs. An other innovative aspect on the proposed device is represented by the study of the appropriate location and amount of the doping polymer, required to improve the sensing capabilities and reduce the costs.

According to their sensing mechanisms [6], polymeric humidity sensors are divided into two fundamental categories: resistive-type and capacitive-type. The former responds to moisture variation by changing its conductivity, while the latter reacts to water vapor by varying its dielectric constant [7]. Among the different polymers showing sensitivity to humidity, one of the most exploited is poly(3,4-ethylenedioxythiophene) : poly (styrenesulfonic acid) PEDOT:PSS [8]. Such a conducting polymer shows significant and reversible modification in its electrical properties when exposed to changing humidity. It can be easily manufactured on an industrial scale and can be used in many deposition techniques [9]. For sensing purposes, it is often deposited into interdigital capacitors and characterized in DC, showing monotonic exponential variation of resistance when exposed to humidity [10]. However, only little knowledge is currently available about the radiofrequency features of the PEDOT:PSS as a sensing material, even without integration with antennas.

The aims of this paper are: 1) to develop a tag geometry suitable to host PEDOT:PSS deposition in a controlled way; 2) to estimate the electromagnetic parameters of the PEDOT:PSS 
in the UHF RFID band for different grades of humidity exposures; 3 ) to characterize the achievable sensitivity, and the communication performance of the sensor tag depending on the amount of deposited polymer.

\section{RFID SENSING EQUATIONS}

Since the two-way RFID reader-tag link is strictly dependent on the variation of local relative humidity $R H^{1}$ [1], it is possible to easily define some sensing indicators directly derivable from the reader's measurements.

A first parameter is the turn-on power $P_{i n}^{t o}[R H]$, e.g. the minimum input power $P_{\text {in }}$ through the reader's antenna required to activate the tag's microchip:

$P_{i n}^{t o}(\theta, \phi)[R H]=\left(\frac{\lambda_{0}}{4 \pi d}\right)^{-2} \frac{P_{c h i p}}{G_{R}(\theta, \phi) \eta_{p} G_{T}(\theta, \phi)[R H] \tau[R H]}$

where $d$ is the reader-tag distance, $G_{R}(\theta, \phi)$ is the gain of the reader antenna, $G_{T}(\theta, \phi)[R H]$ is the gain of the tag's antenna, $\eta_{p}$ is the polarization mismatch between the reader and the tag, $P_{\text {chip }}$ is the IC's sensitivity and $\tau[R H]$ is the power transmission coefficient of the tag :

$$
\tau[R H]=\frac{4 R_{\text {chip }} R_{a}[R H]}{\left|Z_{\text {chip }}+Z_{a}[R H]\right|^{2}}
$$

with $Z_{\text {chip }}$ input impedance of the RFID integrated circuit (IC) and $Z_{A}$ input impedance of the antenna.

From turn-on measurement it is possible to extract the realized gain of the $\operatorname{tag} G_{\tau}[R H]$, e.g. the gain of the tag scaled by the mismatch to the IC, strictly correlated to the physical variation of the local environment:

$$
G_{\tau}(\theta, \phi)[R H]=\left(\frac{4 \pi d}{\lambda_{o}}\right)^{2} \frac{P_{c h i p}}{G_{R}\left(\theta_{R}, \phi_{R}\right) \cdot \eta_{p} \cdot P_{i n}^{t o}(\theta, \phi)[R H]}
$$

By considering the backward link, it is possible to introduce another sensing indicator directly measurable by the reader in term of the Received Signal Strength Indicator (RSSI): the normalized backscattered power $p_{B S}[R H]$, e.g the ratio between the power from the tag to the reader $P_{R \leftarrow T}[R H]$ and the power entering into the reader's antenna $P_{i n}^{t o}$ :

$$
p_{B S}(\theta, \phi)[R H]=\frac{P_{R \leftarrow T}(\theta, \phi)[R H]}{P_{i n}^{t o}(\theta, \phi)[R H]}
$$

\section{The HUMIDITY SENSOR}

The proposed device is a modified version of the wearable antenna presented in [11]: a folded planar structure over a teflon substrate $4 \mathrm{~mm}$ thick, provided with a radiating edge and a sensing $\mathrm{H}$-shaped slot wherein gas-sensitive polymers can be spread (Fig. 1). Since the H-slot acts as an impedance transformer and hosts high value of electric field, the sensitive

\footnotetext{
${ }^{1}$ Relative Humidity $(\mathrm{RH})$ is the ratio of the partial pressure of water vapor present in a gas to the saturation vapor pressure of the gas at a given temperature. It is a function of temperature, and thus it is a relative measurement.
}

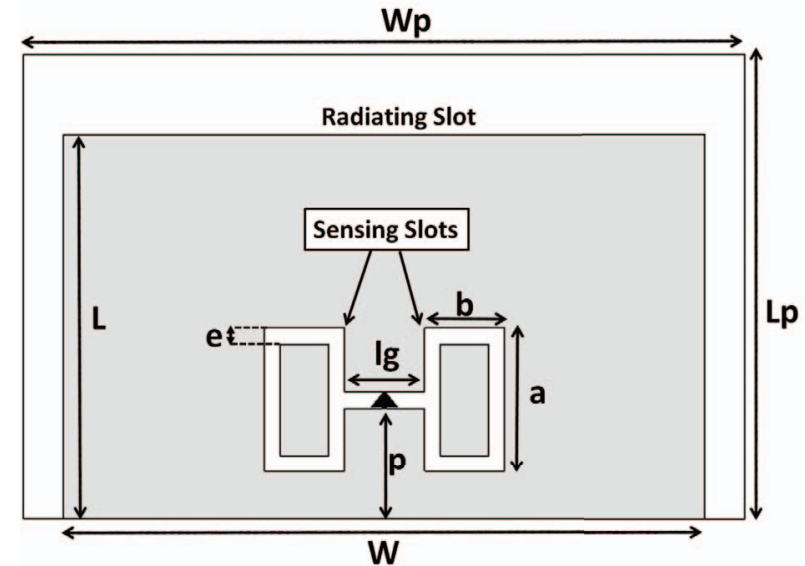

Figure 1. RFID humidity sensor. The PEDOT:PSS polymer will be deposited within the glasses-like slots.

polymers are thus placed just within it [2]. In order to reduce the area of chemical deposition and increase the sensitivity of the device, two rectangular conductive patches have been placed inside the H-slot. In this way, the current is forced to flow with opposite phases along the outer and inner perimeters of the slot and hence each half glasses-like profile may be considered as a combination of six slotlines whose characteristic impedances are affected by the dielectric properties of the polymer spread on top.

The field distribution is not uniform inside the $\mathrm{H}$-slot. The most sensitive area, i.e the portions where the field is maximum, is the central gap (hosting the RFID IC) and the upper inner vertical segments. Since the water absorption produces a change of the polymer's dielectric properties, a variation of both input impedance and losses of the antenna is in turn expected and may be remotely detected by the reader as a modification of the tag's responses.

\section{A. Sensitive material}

The material used in this work is the commercial species Clevios PH 500 [12], a dispersion for conductive coatings with PEDOT to PSS ratio of 1:2.5 (Fig. 2). Like other polymers containing sulfonic acid groups, PEDOT:PSS is strongly hygroscopic and takes up moisture when handled under ambient conditions. Thin layers of PEDOT:PSS films up to a thickness of about $100 \mathrm{~nm}$ are almost able to instantaneously absorb water from the environment: the absorbed water is incorporated into the films and consequently produces an increase of the layer thickness, especially relevent for films with a high PSS content up to $30 \%$ (as in the considered case).

PEDOT:PSS is well known within the antenna and RF community. Thanks to its characteristics, it has been recently used as conductive material for printed antennas applications [13]. Optical translucency of PEDOT:PSS allows to hide an antenna or make the antenna virtually invisible $[14,15]$ when mounted on a transparent substrate, such as the screen of a laptop or PDA, while the ductility allows to attach the polymer on flexible fabrics and plastic substrates. 

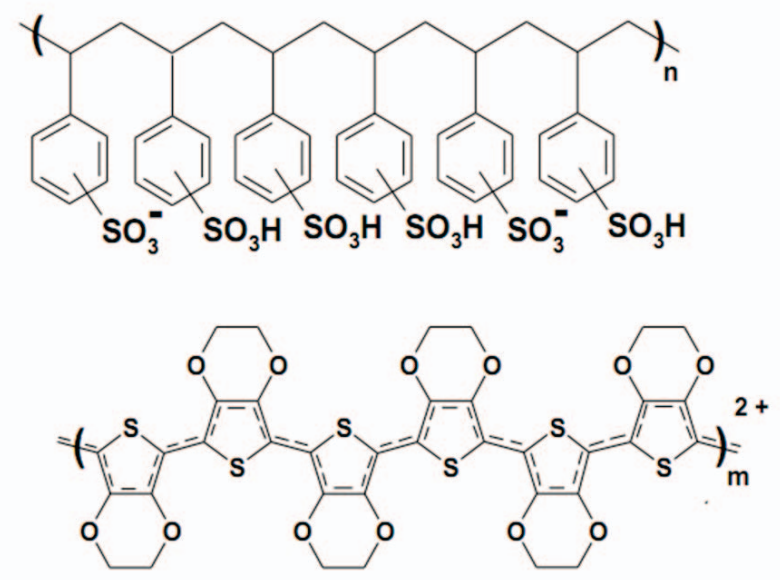

Figure 2. Poly(3,4-ethylenedioxythiophene) : poly (styrenesulfonic acid) PEDOT:PSS chemical formula.

\section{Prototype And Characterization}

A first prototype of the proposed tag, shown in Fig. 3, has been designed and fabricated according to the guidelines described in [11] (size listed in Table I). The tag is matched to the G2iL NXP IC with $Z_{c h i p}=26-j 227 \Omega$ and power sensitivity $P_{\text {chip }}=-18 \mathrm{dBm}$. The design procedure has been optimized for the unloaded tag, i.e. without polymer spread on the H-slot.

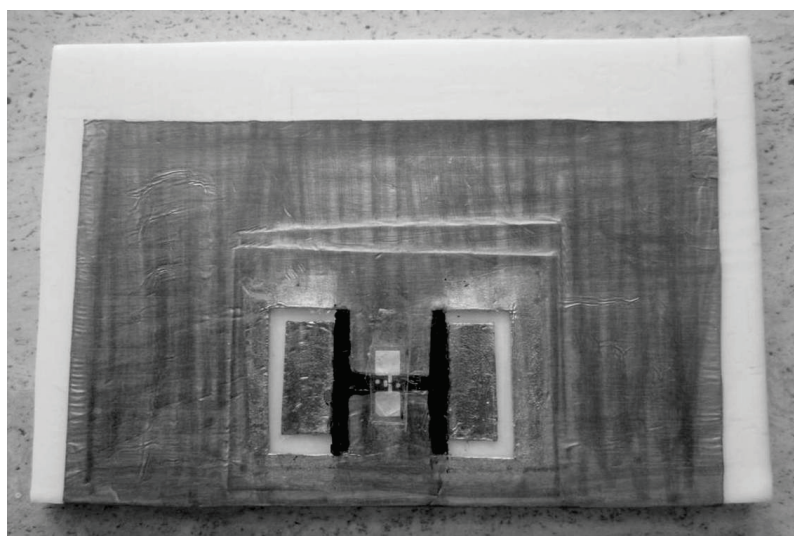

Figure 3. RFID sensor prototype over a Teflon substrate $4 \mathrm{~mm}$ thick with a partial polymer filling on the sensing glasses-like slots.

Table I

SIZE IN [MM] OF THE PARAMETERS IN FIG. 1.

\begin{tabular}{|c|c|}
\hline Parameter & Value $[\mathrm{mm}]$ \\
\hline $\mathrm{a}$ & 18 \\
\hline $\mathrm{b}$ & 10 \\
\hline $\mathrm{e}$ & 2 \\
\hline $\mathrm{p}$ & 14 \\
\hline $\mathrm{L}$ & 48 \\
\hline $\mathrm{Lp}$ & 58 \\
\hline $\mathrm{lg}$ & 10 \\
\hline $\mathrm{W}$ & 80 \\
\hline $\mathrm{Wp}$ & 90 \\
\hline
\end{tabular}

The communication features of the unloaded tag was characterized for what concerns the realized gain $G_{\tau}$ (3) by

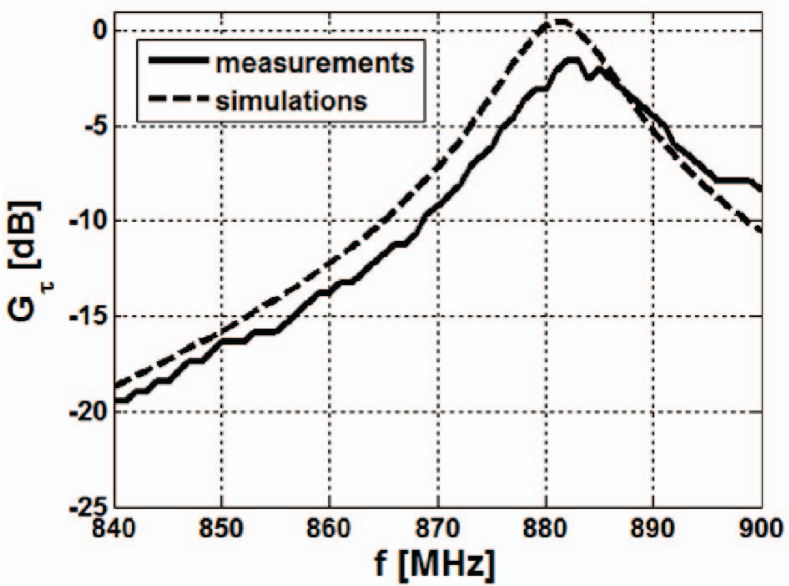

Figure 4. Measured and Simulated realized gain long the antenna axis (broadside observation) for the unloaded sensor tag.

means of both simulations and measurements. Simulations were performed by a Finite-Difference Time-Domain (FDTD) tool. The measurements were carried on by means of a UHF long-range reader based on the ThingMagic M5-e ASIC whose ouput power can be controlled by steps of $0.5 \mathrm{dBm}$. The sensor tag was interrogated by means of a $5 \mathrm{~dB}$ linear polarized patch antenna connected to the reader and placed $50 \mathrm{~cm}$ apart from the tag itself. Ground and walls reflections were minimized by using absorbing panels. The realized gain was hence estimated according to the turn-on method [16]. Results are shown in Fig.4.

From (1) it is possible to determine the maximum activation distance, $d_{\max }=8 \mathrm{~m}$, by considering $3.2 \mathrm{~W}$ EIRP, e.g.the maximum emitted power from the reader allowed by European regulations.

\section{A. PEDOT:PSS Characterization}

While it is possible to provide some electrical information about the PEDOT:PSS in DC [12], no significant data are currently available in the UHF RFID band. In the following analysis the PEDOT:PSS is assumed as a lossy conductor, as described in [13], and its unknown conductivity $\sigma_{P}$ is here identified by the following procedure. The real tag is doped by the polymer as shown in Fig.3 and its realized gain $G_{\tau, f_{i}}^{\text {meas }}[R H]$ is measured at three frequencies $f_{i}=\{860,870,880\}$ in two different humidity conditions: ambient air ( $\mathrm{RH}=50 \%)$ and wet air $(\mathrm{RH}=100 \%)$. The second condition has been obtained by placing the tag inside a closed plastic chamber partially filled with water (Fig. 5). When the chamber is closed, in about one hour the relative humidity approaches $\mathrm{RH}=100 \%$ (wet air), while in case the cover is removed the humidity is that of the ambient air $(\mathrm{RH}=50 \%)$. The measurements have been performed at room temperature $\left(24^{\circ} \mathrm{C}\right)$, to be considered stable all along the process. Then, a numerical model of the tag is arranged by means of an FDTD solver able to calculate the realized gain, say $G_{\tau, f_{i}}^{s i m}\left(\sigma_{P}[R H]\right)$. The conductivity of the material simulating the PEDOT:PSS deposition is therefore optimized in order to minimize, independently for the two humidity grades, the following error 


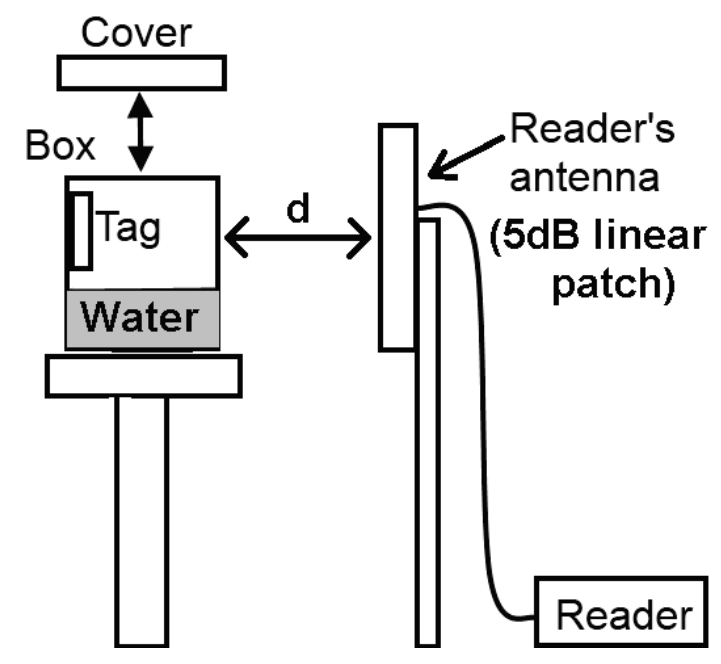

Figure 5. Measurement set-up: the sensor tag is placed into a sealed plastic chamber partially filled with water and $50 \mathrm{~cm}$ away from the reader's antenna. The plastic chamber, thanks to its low permittivity, does not significantly affect the response of the tag in either low and high humidity conditions, as verified by comparing simulations and measurements results.

function $\Delta_{t o t}[R H]$ :

$\sigma_{P}: \Delta_{\text {tot }}[R H]=\sum_{i=1}^{3} \frac{\left|G_{\tau, f_{i}}^{\text {meas }}[R H]-G_{\tau, f_{i}}^{\text {sim }}\left(\sigma_{P}[R H]\right)\right|^{2}}{\left|G_{\tau, f_{i}}^{\text {meas }}[R H]\right|^{2}} \rightarrow 0$

The resulting error profiles for two humidity conditions

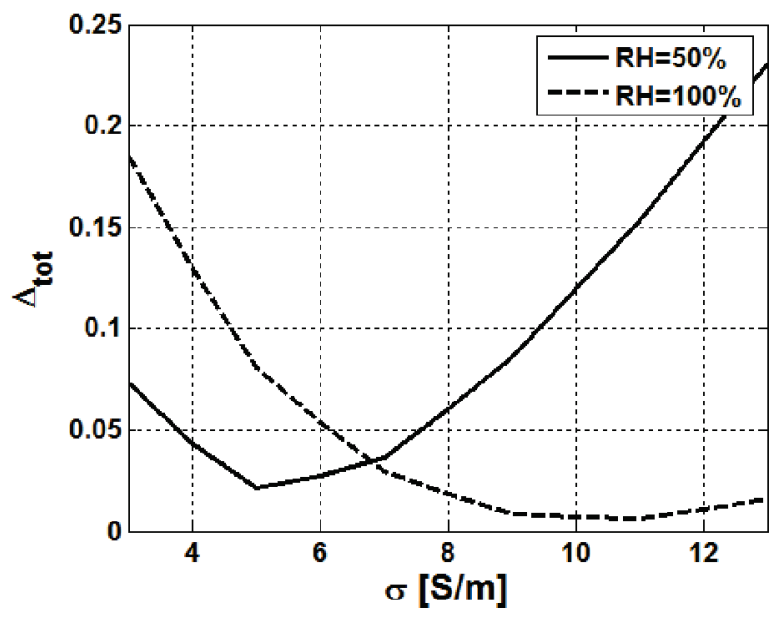

Figure 6. Global error in the parametric identification of the PEDOT:PSS conductivity $\sigma_{P}$ for ambient dry air $(\mathrm{RH}=50 \%)$ and wet air $(\mathrm{RH}=100 \%)$.

( $\mathrm{RH}=50 \%$ and $\mathrm{RH}=100 \%)$ are shown in Fig.6. The estimated values of $\sigma_{P}$ minimizing the global error $\Delta_{t o t}$ are hence $\sigma_{P}(R H=50 \%)=5 S / m$ and $\sigma_{P}(R H=100 \%)=10 S / m:$ the conductivity of PEDOT:PSS approximately doubles going from ambient air to a completely saturated air, sensibly increasing the antenna's losses.

\section{OPERATIVE CHARACTERIZATION}

Since there is no decoupling from the operative and structural point of view between antenna and sensor, the proposed device must be characterized and optimized from both perspectives: the main goal is to enhance its sensing capabilities keeping almost unaffected the communication features or at least to find the most suitable tradeoff between the two constraints.

\section{A. Communication performances}

The communication performances have been statically evaluated, e.g. without exposure to the humidity, in term of normalized realized gain $G_{\tau}$ versus the amount of polymer deposition, as sketched in Fig. 7.

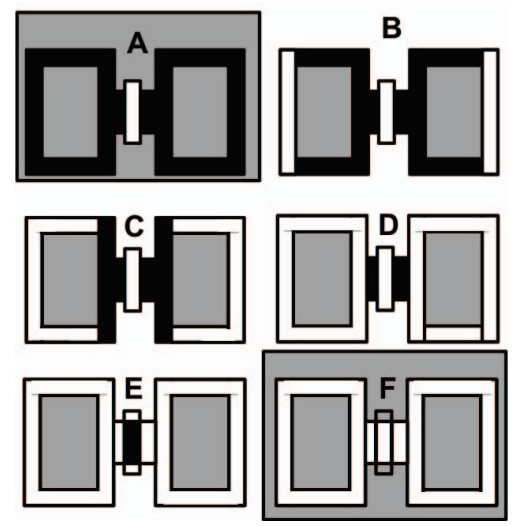

Figure 7. H-slot sensitive area: the sensor tag has been characterized for six different polymer depositions. The first condition (A) corresponds to a complete loading of the slot, the last one (F) to a complete unloaded tag.

The measured profiles, shown in Fig. 8, are normalized with respect to the maximum realized gain of the unloaded tag (case F of Fig.7) as follows:

$$
G_{\tau, \text { norm }}(\xi)=G_{\tau}(\xi)-\max \left(G_{\tau}(F)\right)
$$

whre $\xi=[A, B, C, D, E, F]$ are different depositions of PEDOT_PSS inside the slot (Fig. 7). A nearly $4 d B$ difference of realized gain is clear visible between the completely loaded (A- case) and unloaded (F- case) antenna. As the polymer deposition area increases (from E-case to A-case), slight monotonic variations are measured, such as a frequency shift of about $2 \mathrm{MHz}$ with an attenuation of almost $1 \mathrm{~dB}$. It is worth noticing that even a small drop of PEDOT:PSS (case E) sensibly degrades the gain of about $3 \mathrm{~dB}$, so reducing the expected activation distance from $8 \mathrm{~m}$ to $5.5 \mathrm{~m}$.

\section{B. Sensing features}

The sensing performances of the RFID tag have been dynamically analyzed, i.e. when the humidity inside the box of Fig. 5 gradually changes from ambient conditions up to saturation, versus the amount of polymer deposited into the H-slot.

Fig. 9 shows the measured variation of the turn-on power $P_{i n}^{t o}[R H]$ with respect to the increase of humidity for three different polymer depositions (cases A, C and E in Fig.7). In each case the frequency that guarantees the largest range of variation, i.e the frequency of the minimum turn-on power, has been selected for the measurement. All the configurations 


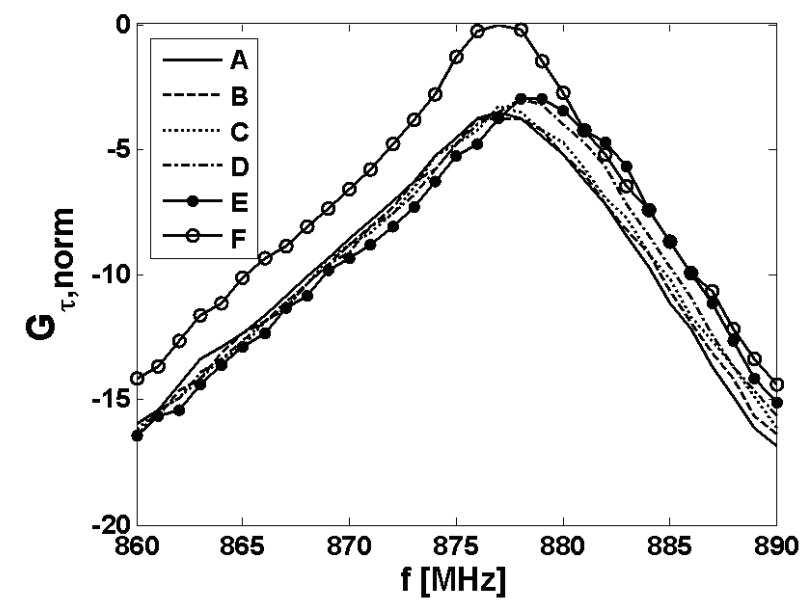

Figure 8. H-slot sensitive area: measured normalized realized gain long the antenna axis (broadside observation).

are suitable to sense the variation of the humidity according to the typical exponential profiles of conventional active sensors. During the exposure process $(t=60 \mathrm{~min}$ sufficient to reach the saturation $\mathrm{RH}=100 \%$ ) the turn-on power increases of about $3.5 d B \div 6 d B$ depending on the specific PEDOT:PSS deposition. The wider is the area covered by the polymer, the greater is the water absorption and thus the variation of the radiation performances of the sensor. The sensor's response indeed saturates for a higher humidity percentage. It is moreover worth noticing that during the early grade of the exposure, e.g. for $50 \%<\mathrm{RH}<75 \%(t<10 \mathrm{~min})$ the slopes of the curves $\mathrm{A}$ and $\mathrm{C}$ are completely overlapped. Hence a similar response may be achieved by using just half the maximum amount of the polymer, with benefit to the possible communication range. Finally, from observation of curve E, it is apparent that even a single drop of polymer placed right under the IC offers the possibility to detect the humidity's change even if at the cost of a smaller sensitivity and dynamic range. The stair-step distribution of the measurement data is due to the limited resolution $(0.5 \mathrm{dBm})$ of the commercial UHF reader.

As for conventional sensor, also in this case it is possible to determine the calibration curves, e.g. the plots of the sensor response versus the variation of the humidity. The normalized values of turn-on and backscattered power respect to the initial condition are visible in Fig.10. The profiles appear almost linear, especially at low humidity grades. The slopes of the curves are in general proportional to the deposition area, except for the early stage of the process. As expected, the curve corresponding to the tag with a single drop of polymer (case E) is the least sensitive one. From Fig. 10 it is possible to extract the sensitivity of the device, e.g the power difference generated by one percent change in the RH level:

$$
S_{\xi}=\frac{|\Delta \xi|}{|\Delta R H|}=\frac{\left|\xi\left(R H_{\text {high }}\right)-\xi\left(R H_{\text {low }}\right)\right|}{\left|R H_{\text {high }}-R H_{\text {low }}\right|}
$$

where $\xi=\left\{P_{i n}^{t o}, p_{B S}\right\}$. The values for the three depositions of Fig. 10 are listed in Table II.

The degree of load and humidity absorption jointly con-
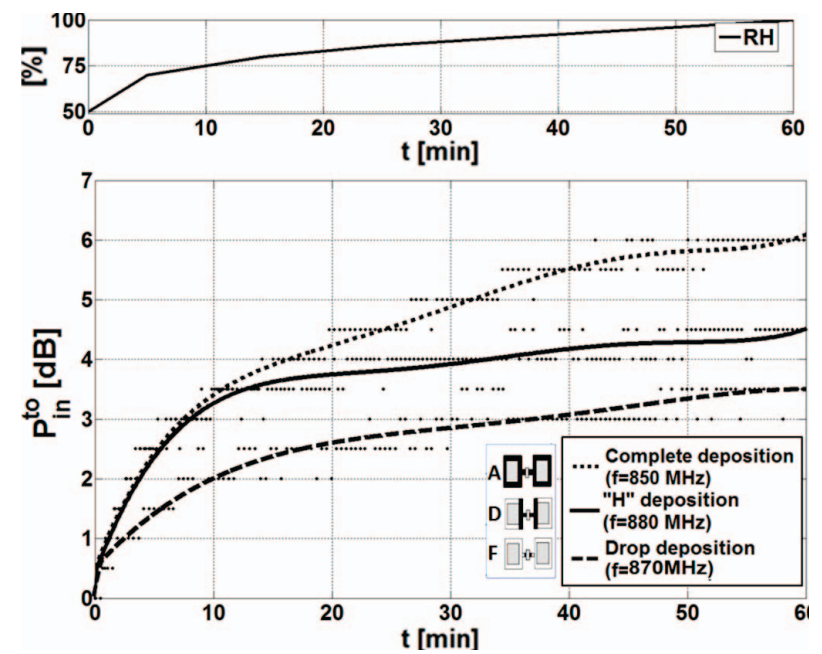

Figure 9. Top) Measured variation of humidity inside the plastic box. The RH level variation with time has been monitored by means of a digital hygrometer placed inside the box. Bottom) Turn-on power normalized with respect to its initial value, e.g at ambient $\mathrm{RH}$, for three different polymer depositions into the tag's glasses-like slots. Measured data (discontinuous dots) have been fitted by a mean square interpolation.

Table II

TURN-ON AND BACKSCATTER SEnsitivities $[d B / R H]$.

\begin{tabular}{|c|c||c||c||c|}
\cline { 2 - 5 } \multicolumn{1}{c|}{} & $R H_{\text {low }}-R H_{\text {high }}$ & Case A & Case C & Case E \\
\hline \multirow{3}{*}{$S_{P_{\text {in }}^{t o}}$} & $50 \%-80 \%$ & 0.13 & 0.12 & 0.08 \\
\cline { 2 - 5 }$S_{p_{B S}}$ & $80 \%-100 \%$ & 0.1 & 0.05 & 0.05 \\
\cline { 2 - 5 } & $50 \%-80 \%$ & 0.18 & 0.2 & 0.12 \\
\cline { 2 - 5 } & $80 \%-100 \%$ & 0.13 & 0.05 & 0.05 \\
\hline
\end{tabular}

tribute to weaken the communication robustness of the proposed device. Depending on the required sensitivity and on the range of humidity it has to be monitored, it is thus necessary to reduce the amount of load to the bare minimum: in fact the smaller the quantity of polymer spread in the sensing slots is, the better the communication performances can be preserved all along the sensing process.

\section{CONCLUSIONS}

The complete integration of PEDOT:PSS into RFID tag has been here reported for the first time aiming to design and test a passive radio-sensor suitable to monitor the quality of the air in term of humidity. Communication and sensing performances have been together analyzed and optimized leading to a fully working prototype whose achievable sensitivity and dynamic range can be controlled by the amount of polymer deposited into the sensing slots. This kind of tag is suited to be attached onto the human body for passive body-centric systems as well as to be embedded into plasters or bendages to remotely monitor the healing grade of wounds.

Future research will consider the characterization of the recovery capability of the sensor, the quantification of the hysteresis in cyclic exposures, and the effective realiability in real world applications. 

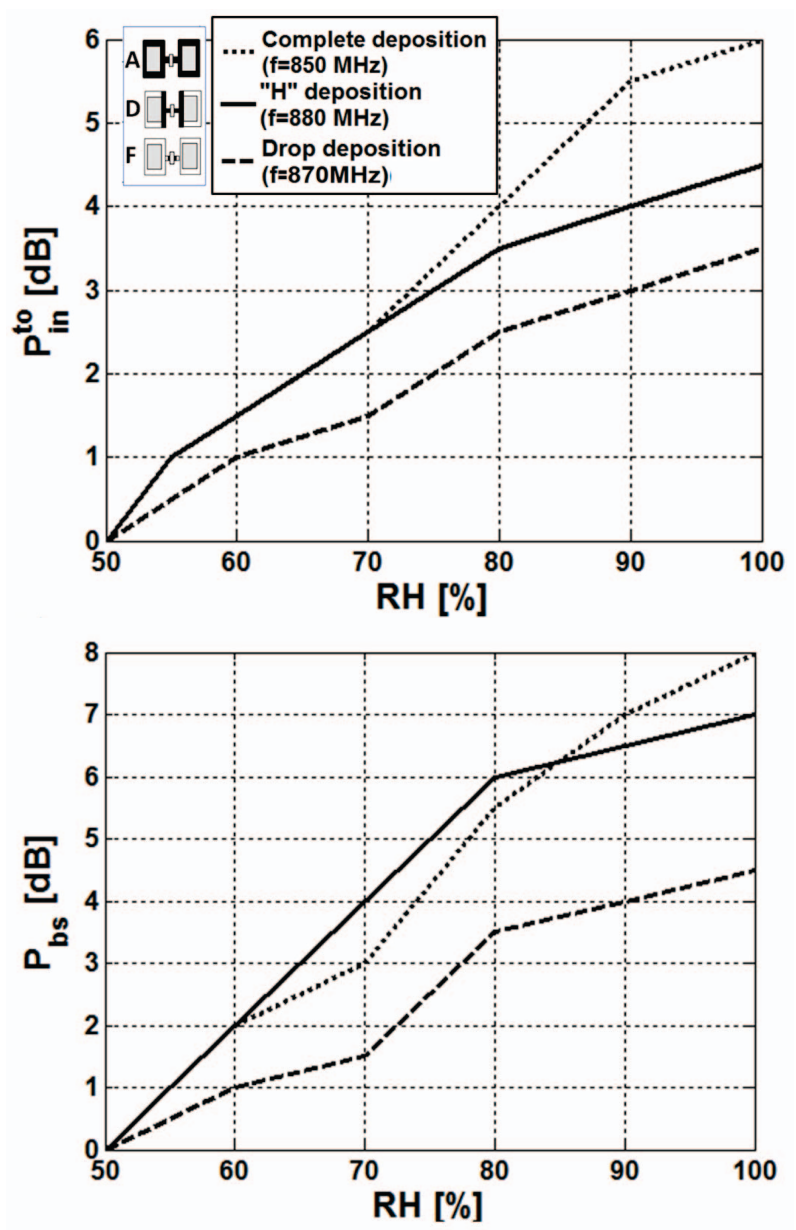

Figure 10. Calibration Curves of the humidity RFID sensor. Top) Turn-on power; Bottom) Backscattered power.

\section{REFERENCES}

[1] G. Marrocco, "Pervasive electromagnetics: sensing paradigms by passive rfid technology," Wireless Cоттиnications, IEEE, vol. 17, pp. 10 -17, december 2010.

[2] C. Occhiuzzi, A. Rida, G. Marrocco, and M. Tentzeris, "Rfid passive gas sensor integrating carbon nanotubes," Microwave Theory and Techniques, IEEE Transactions on, vol. 59, pp. $2674-2684$, oct. 2011.

[3] S. Caizzone, C. Occhiuzzi, and G. Marrocco, "Multichip rfid antenna integrating shape-memory alloys for detection of thermal thresholds," Antennas and Propagation, IEEE Transactions on, vol. 59, pp. 2488 -2494, july 2011.

[4] S. Johan, X. Zeng, T. Unander, A. Koptyug, and H.-E. Nilsson, "Remote moisture sensing utilizing ordinary rfid tags," in Sensors, 2007 IEEE, pp. 308 -311, oct. 2007.

[5] J. Virtanen, L. Ukkonen, T. Bjorninen, A. Elsherbeni, and L. Sydandnheimo, "Inkjet-printed humidity sensor for passive uhf rfid systems," Instrumentation and Measurement, IEEE Transactions on, vol. 60, pp. 2768 -2777, aug. 2011.

[6] H. Bai and G. Shi, "Gas sensors based on conducting polymers," Sensors, vol. 7, no. 3, pp. 267-307, 2007.
[7] Y. Sakai, Y. Sadaoka, and M. Matsuguchi, "Humidity sensors based on polymer thin films," Sensors and Actuators B: Chemical, vol. 35, no. 1-3, pp. 85 - 90, 1996.

[8] A.Elschner, S.Kirchmeyer, W.Lovenich, U.Merker, and K.Reuter, PEDOT: Principles and Applications of an Intrinsically Conductive Polymer. CRC Press, 2010.

[9] T. Kinkeldei, C. Zysset, K. Cherenack, and G. Troster, "A textile integrated sensor system for monitoring humidity and temperature," in Solid-State Sensors, Actuators and Microsystems Conference (TRANSDUCERS), 2011 16th International, pp. $1156-1159$, june 2011.

[10] J. Reboun, A. Hamacek, T. Dzugan, and M. Kroupa, "Sensorial characteristics of conductive polymers," in Electronics Technology, 2009. ISSE 2009. 32nd International Spring Seminar on, pp. 1 -5, may 2009.

[11] C. Occhiuzzi, S. Cippitelli, and G. Marrocco, "Modeling, design and experimentation of wearable rfid sensor tag," Antennas and Propagation, IEEE Transactions on, vol. 58, pp. $2490-2498$, aug. 2010.

[12] "Clevios ph500 http://clevios.com/.".

[13] M. J. J. Kamil, "Flexible temperature sensor integrated with rfid tag," in XIII International PhD Workshop OWD, October 2011.

[14] N. Kirsch, N. Vacirca, T. Kurzweg, A. Fontecchio, and K. Dandekar, "Performance of transparent conductive polymer antennas in a mimo ad-hoc network," in Wireless and Mobile Computing, Networking and Communications (WiMob), 2010 IEEE 6th International Conference on, pp. $9-14$, oct. 2010.

[15] N. Kirsch, N. Vacirca, E. Plowman, T. Kurzweg, A. Fontecchio, and K. Dandekar, "Optically transparent conductive polymer rfid meandering dipole antenna," in RFID, 2009 IEEE International Conference on, pp. 278 -282, april 2009.

[16] G. Marrocco and F. Amato, "Self-sensing passive rfid: From theory to tag design and experimentation," in Microwave Conference, 2009. EuMC 2009. European, pp. 001 -004, 29 2009-oct. 12009. 